\title{
Additive Model to Evaluate the Accuracy of Chest Compression Feedback Systems in Moving Vehicles
}

\author{
Digna M González-Otero ${ }^{1}$, Jesús Ruiz ${ }^{1}$, Sofía Ruiz de Gauna ${ }^{1}$, James K Russell², Luis Leturiondo ${ }^{1}$, \\ Purificación Saiz ${ }^{1}$ \\ ${ }^{1}$ University of the Basque Country (UPV/EHU), Bilbao, Spain \\ ${ }^{2}$ Oregon Health \& Science University (OHSU), Portland, Oregon, USA
}

\begin{abstract}
Quality of cardiopulmonary resuscitation (CPR) affects survival from cardiac arrest. Feedback devices that monitor chest compression rate and depth can be used to guide the rescuer. Many analyze chest acceleration, and could be inaccurate when used in moving vehicles. Our aim was to propose an additive model to evaluate the accuracy of accelerometer-based feedback devices in moving vehicles and to apply it to the case of a plane.

Volunteers provided chest compressions to a resuscitation manikin in static conditions with an accelerometer placed beneath their hands. Dynamic noise was measured during the plane trips Bilbao-Munich and Frankfurt-Bilbao. The acceleration that would have been measured by a feedback device used in a plane was modeled as the sum of the acceleration measured in static conditions and the dynamic noise. Compression depth and rate were estimated from the acceleration both in static conditions and when adding dynamic noise.

In static conditions, median (IQR) unsigned error in depth and rate estimation were $1.4(0.6,2.3) \mathrm{mm}$ and 0.9 $(0.4,1.5) \mathrm{cpm}$, respectively. When adding dynamic noise of the plane, errors were $1.6(0.7,2.9) \mathrm{mm}$ and $0.9(0.4,1.5)$ cpm. The additive model simplifies the evaluation of the accuracy of CPR feedback devices in moving vehicles. In the evaluated conditions, the algorithm was accurate.
\end{abstract}

\section{Introduction}

In the event of cardiac arrest, early and high quality cardiopulmonary resuscitation (CPR) is pivotal for survival [1]. When the patient presents a shockable rhythm, CPR increases the chances of successful defibrillation [2]. CPR has also been associated with higher survival rates even when defibrillation is not required [3].

For optimal outcomes, resuscitation guidelines recommend providing chest compressions with a depth of at least $5 \mathrm{~cm}$ (but no more than $6 \mathrm{~cm}$ ), and with a rate of between 100 and 120 compressions per minute (cpm) [4].
However, meeting these requirements is difficult both for laypeople and for well-trained rescuers [5]. The use of monitoring and feedback devices has been suggested to reduce this problem $[1,6]$.

Many feedback devices are based on accelerometers; they sense the acceleration of the chest during CPR and provide feedback on chest compression depth and rate. These devices could be inaccurate in moving vehicles, in which the acceleration due to the movement of the vehicle would be registered along with that due to the movement of the chest.

In a previous work [7] we evaluated the accuracy of two commercial feedback devices in a long-distance train. For that purpose, we fitted a resuscitation manikin with a displacement sensor and provided chest compressions to the manikin in the train while using the feedback devices. Then we compared the compression rate and depth values computed by both devices with those obtained from the displacement sensor of the manikin, and evaluated the error in the estimation of the feedback parameters for each of the devices. The main drawback of this approach is that the deployment of the experimental set-up in the transportation means is cumbersome, and may even disturb the passengers. The application of this protocol would be particularly challenging on other transportation means such as planes, in which available space is limited.

The aim of this paper was to propose a simplified method to evaluate the accuracy of accelerometer-based feedback devices in moving vehicles. We describe an additive model for the acceleration and apply it to the case of a plane.

\section{Materials and methods}

Our hypothesis was that the acceleration recorded by a feedback device when CPR is provided in a moving vehicle could be modelled as the sum of the acceleration that would be recorded if CPR was provided in static conditions plus the acceleration of the vehicle.

We applied this model to predict the accuracy of an accelerometer-based feedback system in a plane. First, we 
recorded both the acceleration of the chest during CPR in the laboratory (static conditions), and the acceleration of the plane during movement (dynamic noise). Then, we added both accelerations and computed chest compression rate and depth. The accuracy of the method in the plane was assessed by comparing the computed feedback parameters (rate and depth values) with the gold standard obtained from the reference compression depth signal. The process is described in detail in the following sections.

\subsection{Data collection}

\subsubsection{Static measurements}

A Resusci Anne CPR manikin (Laerdal Medical, Norway) was equipped with a photoelectric sensor (BOD 6K-RA01-C-02, Balluff, USA) to measure the reference chest displacement signal. Twenty volunteers provided chest compressions to the manikin in the laboratory (static conditions) with a tri-axial accelerometer (ADXL330, Analog Devices, USA) placed beneath their hands. The reference chest displacement signal and the tri-axial acceleration (static acceleration) were digitized using an acquisition card (USB NI-6211, National Instruments, USA) connected to a laptop computer. The acquisition process was controlled with a custom-made Matlab (Mathworks, USA) program. Signals were acquired with a sampling frequency of $1 \mathrm{kHz}$ and a 16-bit resolution. Figure 1 shows the experimental set-up: the CPR manikin, the acquisition card and the laptop computer (left), the displacement sensor placed inside the manikin (bottom circle), and the tri-axial accelerometer (top circle).

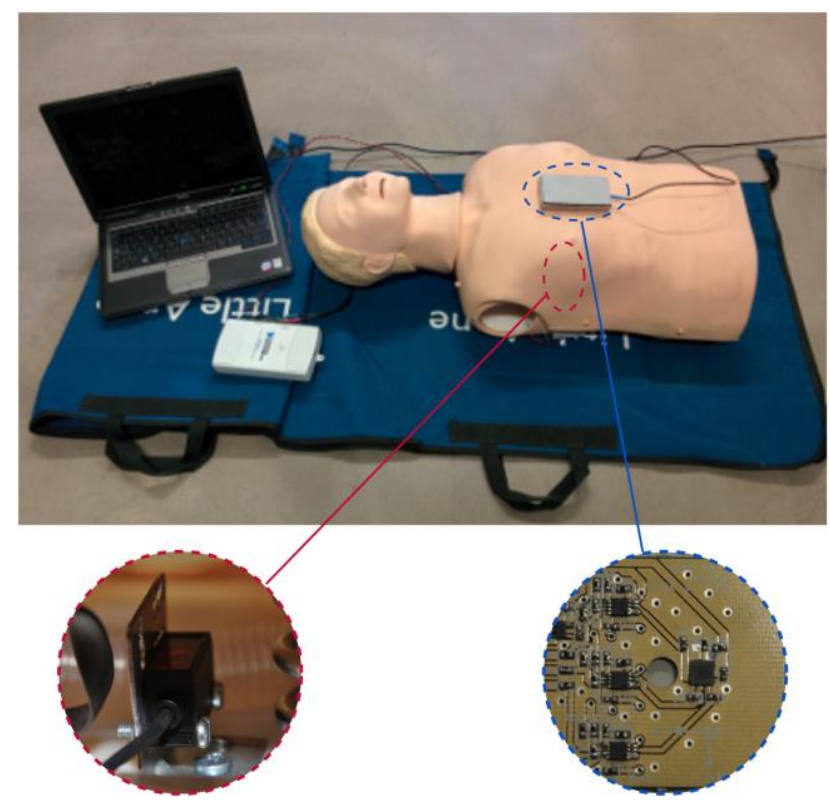

Figure 1. Experimental set-up for static measurements
A total of 20 1-min episodes were acquired, while volunteers provided continuous chest compressions with a target rate of $100 \mathrm{cpm}$ and a target depth of $5 \mathrm{~cm}$.

\subsubsection{Dynamic noise measurements}

Acceleration of the plane was acquired by placing the tri-axial accelerometer described in the previous section on the floor of the plane, connected to the acquisition card and to the laptop computer.

We recorded the acceleration during $60 \mathrm{~min}$ for the trips Bilbao-Munich and Frankfurt-Bilbao, with a sampling frequency of $10 \mathrm{kHz}$.

\subsection{Depth and rate calculation method}

The method [8] we used to compute compression depth and rate from the acceleration signal is based on the idea that, during short time intervals of chest compressions, acceleration and displacement are quasi-periodic. We modelled each 2-s segment of the acceleration and displacement signals using the first 3 harmonics of their Fourier series representation, without DC component.

For that purpose, we first computed the FFT of the windowed acceleration signal and estimated the module and phase of the three first harmonic components of the acceleration. It can be proved analytically that the amplitudes and phases of the spectral components of the compression depth can be computed from the ones of the acceleration, taking into account that acceleration is the second derivative of displacement. From these values, a periodic version of the chest displacement during the analysis window can be reconstructed. Average compression rate for each 2-s analysis window is computed from the fundamental frequency of the acceleration, and average chest compression depth is obtained from the reconstructed chest displacement signal.

\subsection{Data analysis}

Gold standard rate and depth values were computed by applying a peak detector to the reference chest displacement signal acquired during static measurements.

Then, performance of the feedback system was evaluated by comparing gold standard values with those obtained from the calculation method in two conditions: first, in static conditions, applying the method to the triaxial acceleration measured in the laboratory, $a_{s}$; second, with dynamic noise, applying the method to the sum of the static acceleration $a_{s}$ plus the dynamic noise of the plane $a_{d}$. In this second case, dynamic noise was first decimated to $1 \mathrm{kHz}$, and then added to the static measurements axis by axis. The composed acceleration was denoted $a_{t}$. 

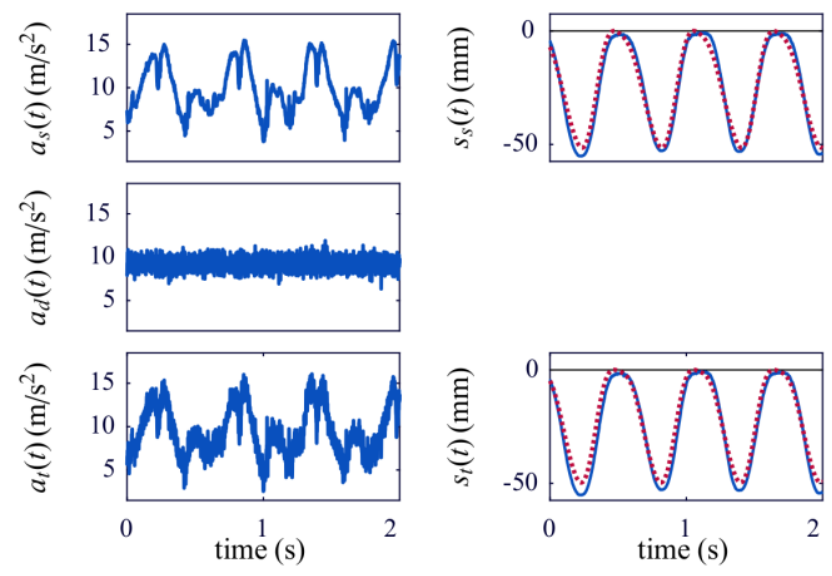

Figure 2. Example of the application of the additive model

Figure 2 shows an example of this procedure. In the top left panel, a 2-second segment of the acceleration recorded during static measurements in the laboratory, $a_{s}(t)$, is shown. On the right, we show the reference compression depth signal acquired by the manikin (solid line), and the computed compression depth signal (dotted line), obtained by applying the depth and rate calculation method to $a_{s}(t)$. For this segment, error in the estimation of compression depth from the acceleration was $0.1 \mathrm{~mm}$, and error in the estimation of compression rate was $0.9 \mathrm{cpm}$. Middle panel shows the dynamic noise recorded in the plane, $a_{d}(t)$. Bottom panel shows the total acceleration $a_{t}(t)$, obtained by adding $a_{s}(t)$ and $a_{d}(t)$ axis by axis. This signal represents the acceleration that would have been measured if chest compressions had been provided on the plane. Bottom right panel shows again the reference compression depth signal (solid line) and the compression depth signal computed from $a_{t}(t)$, represented by the dotted line. In this case error in depth estimation increases to $2 \mathrm{~mm}$, while error in rate estimation remains constant, $0.9 \mathrm{cpm}$.

Distribution of the errors in depth and rate estimation for all the database was analysed using boxplots. Unsigned absolute and relative errors are presented as median (IQR). As results did not pass Lilliefors normality test, Wilcoxon rank sum test was used to perform between-groups comparisons. P-values $<0.05$ were considered significant.

\section{Results}

Figure 3 shows the distribution of the error in depth estimation both in static conditions and with dynamic noise. In static conditions, median (IQR) unsigned error in depth estimation was $1.4(0.6,2.3) \mathrm{mm}, 2.7(1.2,4.4) \%$ of the gold standard depth. With dynamic noise, median unsigned error increased to $1.7(0.7,2.9) \mathrm{mm}, 3.2(1.4$, $5.6) \%$ when expressed in relative units. This difference in errors between static and dynamic conditions was statistically significant $(\mathrm{p}<0.001)$.

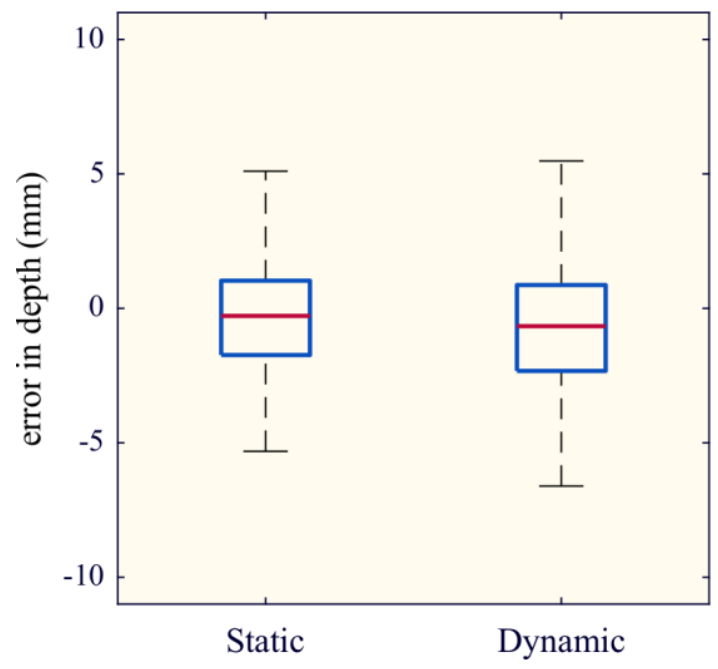

Figure 3. Distribution of the error in depth estimation in static conditions and with dynamic noise.

Figure 4 shows the distribution of the error in rate estimation. In static conditions, median unsigned error was $0.9(0.4,1.5) \mathrm{cpm}, 0.9(0.4,1.5) \%$ of the gold standard rate. When adding dynamic noise, unsigned error did not change significantly $(\mathrm{p}=0.9)$, and had a median of $0.9(0.4$, $1.5) \mathrm{cpm}, 0.9(0.4,1.6) \%$ in relative units.

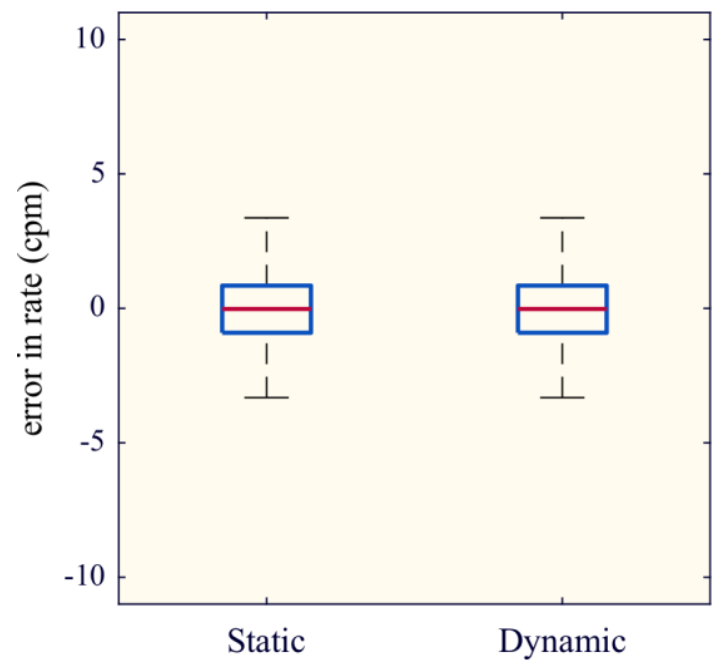

Figure 4. Distribution of the error in rate estimation in static conditions and with dynamic noise.

\section{Discussion}

Early CPR is paramount for survival from cardiac arrest. When cardiac arrest occurs on a long-distance transportation means, such as a ship, a long-distance train or a plane, immediate in situ interventions are required to keep the patient alive until conventional treatment is 
available.

Feedback devices can help rescuers achieve target compression rate and depth during CPR, and thus increase the patient's chances of survival. However, these devices could malfunction and provide inaccurate feedback when used in moving vehicles. Particularly, accelerometer-based devices would record the acceleration of the vehicle along with the acceleration of the chest during chest compressions, which could lead to erroneous depth and rate estimates. Experimental measurements are required to quantify these errors and to decide if they are acceptable before deploying feedback devices in transportation means. In this paper we present a simple method to evaluate the accuracy of acceleration-based CPR feedback devices, based on the additive model of the acceleration.

We applied this method to data acquired in a commercial plane. In the evaluated conditions, noise due to the movement of the plane increased errors in compression depth estimation, but it did not affect compression rate estimation. Even if errors in depth estimation increased, the method was still very accurate, with a median error below $3 \%$ of the target depth, so this feedback device could be safely used in this scenario.

\section{Conclusions}

We presented a novel method to evaluate the accuracy of acceleration-based CPR feedback devices on moving vehicles. To apply this method, the only experimental measurement that needs to be performed in the vehicle is the acquisition of the tri-axial acceleration due to the movement of the vehicle. This method is simple, and could contribute to the safe deployment of CPR feedback devices on board transportation means.

\section{Acknowledgements}

This work received financial support from the Spanish Government through the project TEC2012-31144, from the Basque Government through the grant BFI-2011-166, and through the Vice Cancellor's office for Research of the UPV/EHU through the grant "Contratación de doctores recientes hasta su integración en programas de formación postdoctoral".

The authors would like to thank all colleagues participating in collecting the data.

\section{References}

[1] Meaney PA, Bobrow BJ, Mancini ME, Christenson J, de Caen AR, Bhanji F, et al. Cardiopulmonary Resuscitation Quality: Improving Cardiac Resuscitation Outcomes Both Inside and Outside the Hospital: A Consensus Statement From the American Heart Association. Circulation. 2013;128:417-35.

[2] Waalewijn RA, Nijpels MA, Tijssen JG, Koster RW. Prevention of deterioration of ventricular fibrillation by basic life support during out-of-hospital cardiac arrest. Resuscitation. 2002;54:31-6.

[3] Rea TD, Cook AJ, Stiell IG, Powell J, Bigham B, Callaway CW, Chugh S, Aufderheide TP, Morrison L, Terndrup TE, Beaudoin T. Predicting survival after out-of-hospital cardiac arrest: role of the Utstein data elements. Annals of emergency medicine. 2010;55:249-57.

[4] Perkins GD, Handley AJ, Koster RW, Castrén M, Smyth MA, Olasveengen T, Monsieurs KG, Raffay V, Gräsner JT, Wenzel V, Ristagno G. European Resuscitation Council Guidelines for Resuscitation 2015: Section 2. Adult basic life support and automated external defibrillation. Resuscitation. 2015;95:81-99.

[5] Abella BS, Alvarado JP, Myklebust H, Edelson DP, Barry A, O'Hearn N, Hoek TL, Becker LB. Quality of cardiopulmonary resuscitation during in-hospital cardiac arrest. Jama. 2005;293:305-10.

[6] Krasteva V, Jekova I, Didon JP. An audiovisual feedback device for compression depth, rate and complete chest recoil can improve the CPR performance of lay persons during selftraining on a manikin. Physiological measurement. 2011;32:687.

[7] González-Otero DM, Ruiz de Gauna S, Ruiz J, Chicote B, Rivero R, Russell JK. Accurate feedback of chest compression depth and rate on a manikin in a moving train. Resuscitation. 2015;96:13.

[8] González-Otero DM, Ruiz J, Ruiz de Gauna S, Irusta U, Ayala U, Alonso E. A new method for feedback on the quality of chest compressions during cardiopulmonary resuscitation. BioMed research international. 2014;2014.

Address for correspondence.

Digna M González-Otero

Dpt. Communications Engineering

Faculty of Engineering, UPV/EHU

Alameda Urquijo s/n 48013-Bilbao (Spain)

dignamaria.gonzalez@ehu.eus 